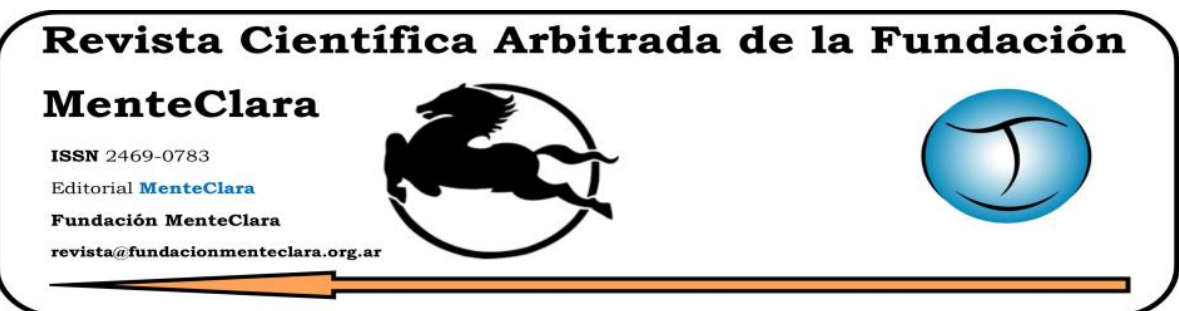

Artículos atravesados por (o cuestionando) la idea del sujeto -y su género- como una construcción psicobiológica de la cultura. Articles driven by (or questioning) the idea of the subject -and their gender- as a cultural psychobiological construction

Vol. 3 (1), 2018

ISSN 2469-0783

https://datahub.io/dataset/2018-3-1-e37

\title{
GUÍA EDITORIAL PARA LA PRESENTACIÓN DE ARTículOS CIENTÍFICOS
}

\section{EDITORIAL GUIDELINES TO WRITE SCIENTIFIC ARTICLES}

Oscar R. Gómez oscar@tantra.org.es

Fundación MenteClara

Cómo citar este artículo: Gómez, O. R. (2018). "Guía editorial para la presentación de artículos científicos". Revista Científica Arbitrada de la Fundación MenteClara, 3(1), 91-101. DOI: 10.32351/rca.v3.1.40

Copyright: ( 2018 RCAFMC. Este artículo de acceso abierto es distribuido bajo los términos de la licencia Creative Commons Attribution-Non Commercial (by-cn) Spain 3.0. Publicación online: 30/4/2018

Conflicto de intereses: Ninguno que declarar.

\section{Resumen}

Este artículo busca ayudar a los autores que deseen presentar sus trabajos en nuestra revista con una presentación acorde con nuestras normas metodológicas y editoriales para asegurarse mayores posibilidades de ser aceptados por los evaluadores externos y por el comité editorial de la Revista Científica Arbitrada de la Fundación MenteClara.

\begin{abstract}
This article aims at helping authors who wish to submit their work to our journal to produce a piece of work written according to our methodological and editorial standards to ensure greater possibilities of being accepted by external evaluators and our journal's editorial committee.
\end{abstract}

\section{Palabras Claves}

Metodología; investigación; clínica; sociología; diagramación; presentación; publicación; artículo científico; Methodology; research; clinical; sociology; layout; presentation; publication; scientific article 


\section{Estructura básica de un artículo de interés científico para nuestra editorial}

Encabezamiento según este mismo artículo y:

\section{Resumen}

Aquí el resumen del artículo de entre 50 y 250 palabras. Que deberá responder las siguientes preguntas:

¿Por qué se realizó?

¿Qué se busca lograr con el trabajo?

¿Cómo se ha hecho?

¿Qué se encontró?

¿Qué importancia tienen los hallazgos?

\section{Abstract}

Aquí el resumen del artículo en inglés.

\section{Palabras Claves}

(En español e inglés) las palabras por las que desea ser encontrado por los motores de búsqueda.

\section{Normas de redacción ${ }^{1}$}

La Revista Científica Arbitrada de la Fundación MenteClara es una revista bilingüe que admite trabajos tanto en español como en inglés. La revista acepta para su publicación:

Artículos o trabajos originales que cumplan con una estructura académica habitual (Sampieri, 2010) en las revistas cientificas: resumen, palabras clave, introducción, exposición de los fundamentos del trabajo y especificación clara de los objetivos; descripción de las fuentes, metodología; exposición de los resultados y discusión de los mismos; conclusiones finales referencias y bibliografia. Se solicita no utilizar notas a pie de página $o$, si son necesarias, recomendamos que se utilice el menor número posible.

\footnotetext{
${ }^{1}$ El uso de pie de página deberá limitarse solo a lo estrictamente necesario como para aclarar algún término, metodología o lo que el autor considere imprescindible para el claro entendimiento de su obra.
} 
Reseñas bibliográficas: se aceptan trabajos destinados a analizar un conjunto de novedades editoriales que por su temática o afinidad puedan dar lugar a un debate o la presentación del estado de la cuestión de un tema específico.

Todos los originales, antes de iniciar el proceso de evaluación por pares, serán leídos previamente por el Consejo Editorial y la Dirección, que especificará la adecuación del manuscrito a las normas y al perfil de contenidos de la revista.

La Dirección de la Revista podrá rechazar un artículo, sin necesidad de enviarlo a los evaluadores externos, si considera que no se adapta a las normas o no se adecua al perfil de contenidos de la publicación.

Los originales que sean revisados seguirán el sistema habitual de "pares", siendo enviado al menos a evaluadores externos a la entidad editora, cuyas sugerencias serán enviadas a los autores para que, en caso necesario, realicen las modificaciones pertinentes.

El método de evaluación empleado es el que se conoce como "doble ciego", manteniéndose el anonimato tanto del autor como de los evaluadores ${ }^{2}$.

El Consejo de Redacción analizará todas las contribuciones y, teniendo en cuenta las evaluaciones externas, decidirá su aprobación o rechazo, así como el volumen y número en el que se publicarán los artículos aceptados. Se informará al autor sobre la aceptación o rechazo de su contribución en un plazo máximo de seis meses.

\footnotetext{
2 Al final de este artículo, en la página 96, encontrará la guía para revisión que emplea la revista. Por favor, antes de enviarnos su artículo, haga usted mismo la revisión para asegurarse un trabajo factible, interesante, novedoso, ético y relevante.
} 


\section{Normas para escritura y envio de artículos}

Por favor utilice este modelo de maquetación para el envío de su trabajo utilizando esta plantilla para el tamaño de hoja, la marginación estilo de letra y párrafos.

-Tamaño de hoja: A4

-Márgenes simétricos. Superior e inferior $2 \mathrm{~cm}$, interior $2 \mathrm{~cm}$ y exterior $1,5 \mathrm{~cm}$.

-Estilo de letra (fuente): Bookman Old Style

-Tamaño de letra: 14

-Alineación: Justificada

-Sangría de primera línea $0,6 \mathrm{~cm}$. Espaciado anterior 6 pto, interlineado sencillo.

(Si tiene dudas, controle la configuración con este artículo que está leyendo.)

\section{Ejemplos de citación APA 6 ta edición:}

Primero deberá cargar las fuentes bibliográficas en Word en la pestaña Referencias $>$ Administrar fuentes $>$ Nueva. Complete los campos del formulario con los datos bibliográficos del documento citado. Una vez en su documento, haga clic al final de la oración o frase que quiera citar y use la opción Insertar cita de la pestaña Referencias para que quede de la siguiente manera:

(Ann Heirman, 2007) (Chhaya, 2009) (Congreso Mundial Uigur, 2004) (Foljambe, 2008) (Gómez, 2013) (Jiménez Rueda, 1945) (Pandit, 1980)

Por último, al final del artículo y en una página en blanco:
A) Vaya a la pestaña Referencias $>$ Bibliografia $>$ Añadir Referencias
B) Vaya a la pestaña Referencias $>$ Bibliografia $>$ Añadir Bibliografia
De este modo, las referencias y la bibliografia se crearán automáticamente al final del artículo siguiendo el formato APA. 


\section{Sugerencia de estructura básica del artículo:}

1. Introducción. Se explica en esta parte el objetivo del artículo, los antecedentes, su estado en la Comunidad Científica y la razón por la que este artículo aporta algo nuevo a la ciencia. La introducción será breve. Utilice las principales palabras clave; las más importantes estarán igualmente en el título y en el resumen.

2. Materiales y métodos. Aquí se explica cómo se ha realizado la investigación, con qué materiales, experiencias, estudios y métodos; dónde y cómo se ha realizado, qué fuentes bibliográficas se han consultado.

3. Resultados. Pueden ser datos, interpretaciones, comentarios. En cualquier caso tienen que ser muy claros y concisos. En general los resultados se presentan en forma de listas, tablas, gráficos e imágenes.

4. Discusión. La discusión estudia la importancia e interpretación de los resultados, comparándolos con otros existentes, y relacionando ese artículo con otros y con otras investigaciones hechas en el mismo contexto.

5. Conclusiones: Es el resumen de la investigación y su estado actual. Aquí se pueden plantear objetivos futuros a seguir en la línea de investigación.

\section{Ejemplo de cómo insertar su trabajo en una plantilla}

Quitele primero el formato al artículo escrito por usted copiándolo en un block de notas sin formato y luego de alli copiarlo y pegarlo dentro del mismo texto en nuestra plantilla de maquetación.

Puede descargar la plantilla de maquetación de:

http://fundacionmenteclara.org.ar/revista/public/journals/1/Plantil laModeloMaquetacion.dotx 
Guia de revisión: A continuación encontrará parte del cuestionario que los revisores deberán responder para evaluar su artículo (Bobenrieth Astete, 1998). Algunas de las preguntas son específicas de determinados marcos académicos. Le sugerimos responder afirmativamente al menos el $50 \%$ de las que corresponden al suyo. Con eso, su artículo no será rechazado y comenzará su proceso de revisión, edición, maquetación y publicación.

¿La relación del título, el contenido y las conclusiones cumplen las normas?

¿El título permite la clara identificación del tema?

¿E1 título recoge las variables de estudio?

¿El resumen evidencia la estructura del artículo?

$¿ E 1$ resumen tiene menos de 250 palabras?

¿El resumen describe el objetivo?

$¿$ ¿El resumen describe el contenido del escrito?

¿En el resumen se expone el problema y el método utilizado en la investigación?

¿Usa denominaciones genéricas de productos farmacéuticos?

¿Las palabras claves identifican el área del conocimiento?

¿Las palabras claves se corresponden con la temática?

¿La introducción sitúa adecuadamente el problema de investigación?

¿La introducción describe la estrategia de investigación?

¿La introducción señala las implicaciones (impacto científico y social) del estudio?

¿La introducción identifica la posición del autor?

¿La introducción desarrolla los antecedentes de la investigación?

¿La introducción enuncia el propósito de la investigación?

¿La introducción enuncia los objetivos de la investigación?

$¿$ En la metodología se exponen los procedimientos utilizados por el autor para el diseño de la investigación? 
¿En la metodologia se justifica la selección de variables y/o de la muestra(s) utilizada(s) en la investigación?

¿En la metodología se describe el enfoque y método de la investigación?

¿En la metodología se describen los instrumentos utilizados para la recolección de la información?

¿En la metodología se exponen los procedimientos utilizados por el autor para el análisis de datos recolectados?

¿La investigación se vincula con teorias existentes?

¿El marco teórico es adecuado para el problema de la investigación?

¿El marco teórico se desarrolla en forma lógica y comprensible?

¿El marco teórico es útil para clarificar los conceptos y las relaciones entre ellos?

¿El artículo especifica la unidad de análisis -caso, serie de casos, muestra o población total-?

¿La población diana se identifica y describe con claridad?

¿La población accesible al estudio se describe con exactitud?

¿El tamaño de la muestra se informa a la luz del objetivo del estudio, el diseño del estudio, el método de muestreo y el análisis estadístico de los datos?

¿La muestra indica cuán representativa es de la población diana a la que se intenta generalizar con los resultados?

¿El método de selección y asignación de sujetos a los grupos de estudio y de control se describe con claridad?

¿Las variables se organizan en grupos lógicos clínicamente: variables de criterios de inclusión, variables factores de riesgo y variables de resultado?

¿Los grupos de estudio y de control son comparables?

¿Se indica con precisión la duración del estudio para ambos grupos (estudio y control)?

¿El artículo tiene coherencia interna (el resumen, la introducción, los objetivos, la metodología y los resultados presentados se complementan e integran adecuadamente)?

¿El artículo cuenta con una base conceptual de fondo que soporte la argumentación?

¿La base conceptual utilizada por el autor para argumentar en su artículo es seria, de actualidad y autoridad en su área de conocimiento? 
¿El artículo de revisión responde a un estudio serio, en detalle y crítico de un tema en particular?

¿El artículo examina bibliografia publicada y reconocida?

¿El artículo registra por lo menos una cita bibliográfica cada dos párrafos?

¿Los resultados son claros?

¿Los resultados se corresponden con los objetivos propuestos?

¿Los resultados se presentan adecuadamente para una fácil comprensión de los lectores?

¿Los resultados derivan directamente del análisis de los datos recolectados?

¿Los resultados contribuyen a la solución del problema planteado?

¿Las conclusiones presentadas aportan nuevos conocimientos teóricos o prácticos sobre la temática trabajada?

¿El autor asume una posición sobre el tema de investigación?

¿El artículo es factible?

$¿ E 1$ artículo es interesante, no posee contenido que el lector ya debe conocer?

¿El artículo y la metodología de estudio siguen normas éticas adecuadas?

¿Se describe el procedimiento para obtener consentimiento informado?

¿Hay constancia de la revisión de la investigación por algún consejo o comité de ética?

$¿$ El investigador describe los riesgos potenciales de los sujetos participantes del estudio?

¿Hay constancia de que se aseguró el anonimato y la confidencialidad a los participantes del estudio?

¿Se proponen investigaciones a futuro sobre el problema planteado en el artículo y basados en la experiencia lograda durante la investigación presentada?

¿Las citas textuales siguen el modelo de APA?

¿Las referencias bibliográficas siguen el modelo de APA?

¿Las referencias citadas en el texto están bien documentadas y son actuales? 


\section{Ejemplo de formato de referencias}

\section{Referencias}

Ann Heirman, S. P. (2007). The Spread of Buddhism. Boston: Zürcher Oberland. Obtenido de https:// books.google.es /

Bobenrieth Astete, M. A. (1998). Escritura y lectura critica de artículos científicos. En R. Burgos Rodríguez (ed.), Metodología de investigación y escritura científica en clínica. Parte IV. Granada: Escuela Andaluza de Salud Pública.

Capra, F. (2000). El Tao de la Física. Sirio.

Chhaya, M. (2009). Dalai Lama. Hombre, monje, místico. Grijalbo.

Congreso Mundial Uigur. (2004). Breve historia de Turkestán Oriental. Alemania: Primera Asamblea General del WUC. Obtenido de http://www.uyghurcongress.org/es/?cat=132

de Mora Vaquerizo, J. M. (1988). Tantrismo Hindú y Proteico. Universidad Nacional Autónoma de México.

Foljambe, A. (2008). Tantric Buddism, desire, and the bodyin surrealism and Georges Bataille. University of Manchester.

Gómez, O. R. (2013). El tantrismo dentro de la Compañía de Jesús - del Tíbet al Vaticano hoy (Primera ed.). (F. MenteClara, Ed.) Buenos Aires, Argentina: Ediciones Menteclara. Recuperado el 1 de 11 de 2015

Jiménez Rueda, J. (1945). La secta de los alumbrados en la Nueva España (Vols. Tomo XVI, Numeral 1). Mexico: Boletín del Archivo General de la Nación.

Pandit, M. P. (1980). Kularnava Tantra. Rito de las cinco cosas prohibidas. Editorial Eyras.

Sampieri, R. H. (2010). METODOLOGÍA de la investigación. Mexico: McGRAW-HILL / INTERAMERICANA EDITORES. 


\section{Ejemplo de formato de bibliografia}

\section{Bibliografía}

Ann Heirman, S. P. (2007). The Spread of Buddhism. Boston: Zürcher Oberland. Obtenido de https:// books.google.es/

Bobenrieth Astete, M. A. (1998). Escritura y lectura critica de artículos científicos. En R. Burgos Rodríguez (ed.), Metodología de investigación y escritura científica en clínica. Parte IV. Granada: Escuela Andaluza de Salud Pública.

Capra, F. (2000). El Tao de la Física. Sirio.

Chhaya, M. (2009). Dalai Lama. Hombre, monje, místico. Grijalbo.

Congreso Mundial Uigur. (2004). Breve historia de Turkestán Oriental. Alemania: Primera Asamblea General del WUC. Obtenido de http://www.uyghurcongress.org/es/?cat=132

de Mora Vaquerizo, J. M. (1988). Tantrismo Hindú y Proteico. Universidad Nacional Autónoma de México.

Foljambe, A. (2008). Tantric Buddism, desire, and the bodyin surrealism and Georges Bataille. University of Manchester.

Gómez, O. R. (2013). El tantrismo dentro de la Compañía de Jesús - del Tíbet al Vaticano hoy (Primera ed.). (F. MenteClara, Ed.) Buenos Aires, Argentina: Ediciones Menteclara. Recuperado el 1 de 11 de 2015

Jiménez Rueda, J. (1945). La secta de los alumbrados en la Nueva España (Vols. Tomo XVI, Numeral 1). Mexico: Boletín del Archivo General de la Nación.

Pandit, M. P. (1980). Kularnava Tantra. Rito de las cinco cosas prohibidas. Editorial Eyras.

Sampieri, R. H. (2010). METODOLOGÍA de la investigación. Mexico: McGRAW-HILL / INTERAMERICANA EDITORES. 


\section{Referencias}

Sampieri, R. H. (2010). METODOLOGÍA de la investigación. Mexico: McGRAW-HILL / INTERAMERICANA EDITORES.

Bobenrieth Astete, M. A. (1998). Escritura y lectura critica de artículos científicos. En R. Burgos Rodríguez (ed.), Metodología de investigación y escritura científica en clínica. Parte IV. Granada: Escuela Andaluza de Salud Pública.

Otras fuentes: Revista e-Ciencias de la Información. Escuela de Bibliotecología y Ciencias de la Información, Universidad de Costa Rica San José 2060, Costa Rica. Tel. (506)2511-1919 - Fax: (506) 25111949 http://revistas.ucr.ac.cr/index.php/eciencias/index 